\title{
COMPARISON OF A DAILY AND ALTERNATE-DAY DUAL DRUG REGIME OF CHEMOTHERAPY IN THE TREATMENT OF PULMONARY TUBERCULOSIS
}

\author{
Dr. A. W. T. EADE, B.Sc., M.B., M.R.C.P., D.Phys.Med.* \\ Brigadier JOHN MACKAY-DICK, O.B.E., M.B., F.R.C.P.***
}

\section{Introduction}

We have described previously the radiological and bacteriological response in pulmonary tuberculosis to a regime of chemotherapy in which streptomycin and isoniazid were given together on alternate days following a preliminary period of daily therapy (Mackay-Dick, 1954, 1958, 1963b, 1963c and 1965, Lancet, 1958, Eade et al, 1959, Eade, Harrison, Large and Mackay-Dick, 1968). It is our purpose now to describe a similar study in which the same drugs were used but given daily, and to compare the results of treatment with these two regimes of chemotherapy.

\section{Method \\ Grouping of patients}

The patients were all young Gurkha soldiers; the two groups were therefore matched for race, age and sex. The drugs used were streptomycin $1.0 \mathrm{~g}$ given by intramuscular injection and isoniazid, two $100 \mathrm{mg}$ doses orally, on each treatment day. There were 95 patients in the group treated with the alternate-day regime and 52 in the group treated with the daily regime. In the 'alternate-day' group the period for which chemotherapy was given ranged from 9 to 28 months (average 18 months); in the 'daily' group the range was 7 to 22 months (average 15 months). The duration of chemotherapy for the patients of both groups is compared in Table 1. All of the patients were treated in hospital and the management of the two groups differed only in respect of the regime of chemotherapy.

\section{Radiological assessment}

The extent of the disease present on admission was classified in the manner suggested by Foster-Carter et al (1952). The findings for the two groups are presented in Table II and show that they were reasonably comparable in this respect. In the 'alternate-day' group 55 patients (58 per cent) had cavitated disease and in these a total of 67 cavities were present. In the 'daily' group 20 patients ( 38.5 per cent) had cavitated disease; in these 21 cavities were seen. The proportion of large cavities (diameter greater than $2.5 \mathrm{~cm}$ ) was higher in the 'daily' group (Table III).

\section{Results}

\section{Radiological behaviour during chemotherapy}

We judged radiological stability to have been reached when three consecutive radiographs, taken at six-week intervals, had shown no change. The radiological changes

*Formerly officer-in-charge Medical Division, Connaught Hospital (Army Chest Centre). Present Address : Coventry \& Warwickshire Hospital, Coventry.

**Formerly Commanding Officer, Connaught Hospital (Army Chest Centre), Bramshott and British Military Hospital, Kinrara. Present Address: Headquarters, Scottish Command. 
observed during treatment are summarised and compared for the two groups in Table IV. Some differences were apparent in the behaviour of the disease in the two groups.

Table I

Duration of chemotherapy in the two groups

\begin{tabular}{lcrc}
\hline Duration of chemotherapy & $\begin{array}{c}\text { Alternate-day regime } \\
\text { *Number/approximate } \\
\text { percentage }\end{array}$ & $\begin{array}{c}\text { Daily regime } \\
\text { Approximate } \\
\text { percentage }\end{array}$ \\
Less than 9 months & NIL & 1 & 2 \\
9 to 12 months & 11 & 17 & 32 \\
12 to 15 months & 34 & 21 & 40 \\
15 to 18 months & 26 & 5 & 10 \\
Over 18 months & 27 & 8 & 16 \\
\hline
\end{tabular}

* Three of the 95 patients who received alternate-day therapy underwent bilateral partial lung resection. Their chemotherapy continued unchanged between the two operations; each of these is recorded as two cases making a total of 98 .

Table II

Radiological extent of disease on admission

\begin{tabular}{lcccc}
\hline & \multicolumn{2}{c}{ Alternate-day regime } & \multicolumn{2}{c}{ Daily regime } \\
Extent of disease & Number & Percentage & Number & Percentage \\
One zone & 57 & 60 & 32 & 61.5 \\
Two or three zones & 33 & 34.5 & 16 & 31 \\
Four or more zones & 5 & 5.5 & 4 & 7.5 \\
\hline
\end{tabular}

Table III

Size of cavities in the two groups

\begin{tabular}{lcccc} 
& Alternate-day regime & \multicolumn{2}{c}{ Daily regime } \\
Cavity size (diameter) & Number & Percentage & Number & Percentage \\
Small (under $1.5 \mathrm{~cm})$ & 50 & 64.5 & 10 & 47.5 \\
Medium $(1.5$ to $2.5 \mathrm{~cm})$ & 6 & 9 & 2 & 9.5 \\
Large (over $2.5 \mathrm{~cm})$ & 11 & 16.5 & 9 & 43 \\
\hline
\end{tabular}

Table IV

Radiological behaviour of disease during prolonged chemotherapy

\begin{tabular}{lcccc}
\hline & \multicolumn{2}{c}{ Alternate-day regime } & \multicolumn{2}{c}{ Daily regime } \\
Observations & Number & Percentage & Number & Percentage \\
Stability reached within six months & 20 & 21 & 3 & 5.8 \\
$\begin{array}{l}\text { Stability reached within twelve months } \\
\text { No change during period of }\end{array}$ & 61 & 64 & 24 & 46 \\
$\begin{array}{l}\text { chemotherapy } \\
\begin{array}{l}\text { Stability not reached at time of } \\
\text { operation }\end{array}\end{array}$ & 6 & 6.3 & 11 & 21 \\
Radiological deterioration & 8 & 8.4 & 13 & 29 \\
\hline
\end{tabular}

Radiological change was still occurring at the time of operation in a higher proportion of the patients treated with the daily regime. Two factors probably accounted for this; first, there were six patients in this group who came to surgery before they had completed one year of chemotherapy, whereas there was only one such patient in the group on the alternate-day regime; second, there was a slightly higher incidence of more widespread disease in the group on the daily regime (Table II) and in these circumstances radiological stability unusually takes longer to be achieved.

The cavity closure rate at nine months was 71 per cent in the 'daily' group and 83 
per cent in the 'alternate-day' group. In both groups five cavities persisted throughout the period of chemotherapy, that is less than 8 per cent of the total number of cavities initially present in the ' alternate-day' group, compared with more than 23 per cent in the ' daily' group. In both groups it was the large cavity which persisted or was slow to close and the difference in incidence of persistent cavities was largely a reflection of the different incidence of large cavities in the two groups.

The proportion of patients with non-cavitated disease, showing no radiological change during the time for which they were treated was higher ( 21 per cent) in those on the daily regime than in the 'alternate-day' group (6.3 per cent). The difference in the duration of chemotherapy was probably partly responsible for this. We have noted previously (Eade et al, 1959) that on occasion considerable radiological change in noncavitated disease occurred after 6 to 12 months despite little or no change before that time. In those on the 'alternate-day' regime the duration of chemotherapy ranged from 9 to 28 months (average 15 months) whereas for the 'daily' group it was significantly less, ranging from 7 to 16 months (average 10 months).

In each group one patient showed radiological deterioration. In the patient on alternate-day regime this consisted of cavitation of a previously 'solid' lesion. This event appeared of little moment since sputum and laryngeal-swab culture remained negative, and organisms could not be cultured from the specimen of the lung. The patient receiving daily therapy showed radiological extension of the disease and resistant organisms were cultured from laryngeal swabs, gastric washings and the resected lung.

\section{Bacteriology of the resected specimens}

From the group on alternate-day chemotherapy 98 resected specimens were studied. Positive cultures were obtained from nine; the organisms were resistant to the drugs in use in two of these. The persistence of viable drug-sensitive organisms decreased with increasing duration of chemotherapy (Table V).

Table V

Bacteriology of resected specimens related to duration of alternate-day chemotherapy

Duration of chemotherapy

9 to 12 months

12 to 15 months

15 to 18 months

Over 18 months

Percentage of specimens from which drug sensitive organisms recovered

18

9

8

NIL

From the 52 specimens of lung resected from patients receiving daily chemotherapy a total of 242 samples (from 121 lesions) were examined at the Institute of Diseases of the Chest, and 52 samples (one pooled sample from each specimen) were examined at the Connaught Hospital.

At the Institute of Diseases of the Chest 156 samples were smear-positive and 8 were culture-positive, At the Connaught Hospital 36 samples were smear-positive and one was culture-positive. The positive cultures in the two laboratories related to the same specimen (case 106) and this was also the only one which gave a positive result on guinea pig inoculation. 
Case history 106. This patient was admitted following a routine mass miniature radiograph which showed shadowing in the upper zones of both lungs with a small $(1.0 \mathrm{~cm}$ in diameter) cavity in the right upper lobe. No acid/alcohol-fast bacilli were seen in the sputum and cultures were negative. He was treated with streptomycin $1.0 \mathrm{~g}$ daily and isoniazid $100 \mathrm{mg}$ b.d. The radiographs showed progressive improvement for four months, then the shadowing in the right upper zone extended and the cavity increased in size. Sputum cultures remained negative and no change was made in the chemotherapy at that time. Fourteen months after the chemotherapy had been started he was transferred to the Connaught Hospital where he was given para-aminosalicylic acid (PAS) $12.0 \mathrm{~g}$ daily in addition to streptomycin and isoniazid. Cultures from laryngeal swabs and gastric washings taken on arrival at the Connaught Hospital grew Mycobacterium tuberculosis which proved resistant to streptomycin and partially resistant to isoniazid. Resection of the apical and posterior segments of the right upper lobe was performed. Following operation he developed a broncho-pleural fistula. Streptomycin and PAS were replaced by pyrazinamide, the daily dose of isoniazid was increased to $400 \mathrm{mg}$ and a Robert's flap operation was performed; his further progress was uneventful.

At the Institute of Diseases of the Chest cultures were positive from all of the eight samples examined. The organisms cultured from the cavity and cavity wall proved resistant to streptomycin, partially resistant to isoniazid and sensitive to PAS. Cultures from four samples taken from caseous foci near the cavity proved resistant to streptomycin but sensitive to both isoniazid and PAS. At the Connaught Hospital cultures from the sample proved resistant to streptomycin, partially resistant to isoniazid and sensitive to PAS.

\section{Resistance}

Resistant strains were isolated from three patients in the immediate pre-operative period and from the lung specimens resected from them. All three had cavities still present at the time of operation. Two of the patients had been on alternate-day therapy, thus the incidence of resistance in both groups was approximately 2 per cent. There was no information concerning the sensitivity of the organisms on admission or in the early stages of the illness of these three patients. The possibility of initial infection with resistant strains exists, since this is a common hazard in the countries where these patients contracted tuberculosis. The initial radiological improvement cannot be regarded as evidence that the organisms were then fully sensitive to the drugs since Devadatta et al (1961) have reported patients in whom, following the initial isolation of isoniazidresistant strains, treatment with isoniazid and PAS in combination, and with isoniazid alone, resulted in improvement.

\section{Discussion}

Reports from the Tuberculosis Chemotherapy Centre, Madras (1960, 1963 and 1964) and the Medical Research Council (1962) have suggested that effective continuation therapy for pulmonary tuberculosis does not necessarily involve the administration of anti-tuberculosis drugs every day. Provided that the organisms are sensitive, streptomycin and isoniazid is probably the best combination of drugs (Mitchinson, 1965), and this combination given together at intervals less frequently than daily has been shown to be effective both experimentally (Grumbach, Rist and Riebel, 1952) and clinically (Tuberculosis Chemotherapy Centre, Madras, 1963). The possible advantages of an 
effective alternate-day regime have been referred to previously (Mackay-Dick, 1954, 1960 and 1962, Eade, Harrison, Large and Mackay-Dick, 1968) and recently Poole and Stradling (1965) have reported that these advantages have apparently been achieved by using streptomycin and isoniazid together on alternate days. Although the final assessment of any chemotherapy regime must depend upon the long-term follow-up of treated patients, some indication of its efficacy may be obtained from the clinical and radiological progress whilst it is being given, and, when the opportunity exists, from bacteriological study of resected lung specimens. There was no apparent major difference in the clinical progress of the patients receiving alternate-day therapy and those receiving daily therapy. In both groups the only patients who did not achieve sputum conversion were those with resistant organisms, that is approximately 2 per cent of both groups. There was also no major difference in respect of radiological progress; in both groups just over two thirds of the patients achieved radiological stability within twelve months, although in the group on daily therapy this was in part due to the larger number of patients in whom there was no radiological change at all during treatment. The rate of cavity closure at nine months was better in the 'alternate-day' group ( 83 per cent) than in those on daily therapy (71 per cent). Radiological deterioration occurred in one patient in each group. In the patient on alternate-day therapy this consisted of cavitation of a previously 'solid' nodule, and the event seemed of little significance since there was no extenision of the disease, the sputum remained negative, and organisms could not be recovered from the resected lung. In the patient receiving daily therapy there was radiological extension of the disease after four months treatment, and drug resistant organisms were later cultured from laryngeal swabs and from the resected lung specimen.

With the alternate-day regime positive cultures were obtained from nine of the 98 resected specimens, whilst with the daily therapy group positive cultures were obtained from only one of the 52 specimens. The culture positive specimen from the 'daily' group and two of those from the 'alternate-day' group contained drug resistant organisms. The evidence from bacteriological study of the specimens points to eighteen months of alternate-day chemotherapy being adequate to ensure sterilisation of the lung lesions in the absence of resistance. With daily chemotherapy it appears that, provided drug resistance does not exist, the duration of treatment necessary to achieve this is much less, possibly under one year.

There has been only one report of long-term follow-up of patients treated with this alternate-day regime without surgery (Large and Dimond, 1964) which described five relapses in 78 patients over a five year period. This study was retrospective, the patients having been selected from those treated at the British Military Hospital, Kinrara, before surgical facilities were available. The chemotherapy for these patients differed from that given to the alternate-day group reported here in two potentially important respects; firstly some of the patients were given streptomycin and PAS rather than streptomycin and isoniazid, secondly a higher proportion of the patients were given the short (thirty day) initial period of daily therapy ( 83 per cent compared with 55 per cent of the present group) despite the fact in many of them the extent and nature of the disease was such that this would not have been so if they had been treated a few years later. On the evidence available it is difficult to assess the influence of difference in the duration of the initial period of daily therapy; however, the higher incidence of positive cultures from the 'never-cavitated' specimens, where this period was usually thirty days, than 
from the 'originally-cavitated' specimens, where the period was more often sixty to ninety days, suggests that this may be important. Furthermore, the chemotherapy given to one of the patients whose disease relapsed departed considerably from the standard alternate-day regime and was continued for such a relatively short period (fifteen months) that this case cannot be regarded as a failure of the regime. Also, in none of these cases were drug sensitivities assessed before or during treatment (Mackay-Dick, 1965).

Nevertheless, judged by these long-term follow-up results trea ment with this regime appears less efficient than might be expected with modern regimes of daily chemotherapy. On the other hand, the evidence from bacteriological examination of the resected specimens, which suggests that it is efficient but takes longer to sterilise the lesions than does daily therapy, the excellent clinical and radiological progress during the period of chemotherapy and the absence of significant resistance problems, suggests that with minor modification of the regime better long-term results might have been expected. Although we did not do so there are advantages to giving the isoniazid in a single dose (Gangadharam et al, 1961) and in higher dosage (Mackay-Dick, 1958, Tuberculosis Chemotherapy Centre, Madras, 1963). An alternate-day regime involving these modifications and a routine initial three month period of daily chemotherapy has recently been used by Poole and Stradling (1965) who reported favourably on the short-term findings.

\section{Summary}

Two groups of patients of the same race, sex and age group and suffering from pulmonary tuberculosis of comparable severity were treated with different regimes of long-term continuation therapy. One group of 52 patients received streptomycin $1.0 \mathrm{~g}$ and isoniazid $200 \mathrm{mg}$ (in two $100 \mathrm{mg}$ doses) daily; in the other group the same drugs were given together to 95 patients in similar doses on alternate days after an initial period of daily therapy (Mackay-Dick, 1963b and 1963c). Because of the special circumstances of the patients the residual disease was eventually resected. The resected specimens of lung were examined bacteriologically. The clinical and radiological progress of the two groups was in general similar; in about two thirds of each group there was no further radiological change after twelve months therapy; the cavity closure rate at nine months was 71 per cent in the daily group and 83 per cent in the alternate-day group. In each group radiological deterioration occurred in one patient.

The incidence of resistance was approximately 2 per cent in each group. In the daily-therapy group, apart from one case in which drug resistance occurred, $M$. tuberculosis could not be cultured from the resected specimens despite the fact that four had persisting cavities and 18 had been treated for less than one year. In the alternate-daytherapy group $M$. tuberculosis was cultured from nine of the 98 resected specimens; provided drug resistance did not exist organisms could not be cultured when treatment had been given for longer than eighteen months ( 26 cases). Although judged by clinical and radiological criteria the daily and alternate-day regimes appear to have comparable efficacy, the bacteriological findings suggest that this alternate-day regime takes longer to sterilise the lung lesions. Nevertheless, the surgical results of cases treated on this regime are excellent (Lancet, 1960, Mackay-Dick, 1963a).

\section{Acknowledgements}

We wish to thank Dr. R. W. Riddell and Dr. Lynne Reid under whose direction the 
bacteriological studies at the Institute of Diseases of the Chest were carried out.

\section{REFERENCES}

Devadatta, S., Bhatia, A. L., Andrews, R. H., Fox, W., Mitchison, D. A., Radhakrishna, S., Ramakrishnan, C. V., Salkon, J. B. and Velus, S. (1961) Bull. Wld. Hith Org. 25, 807.

Eade, A. W., HaRrison, G. K., LARGe, S. E., MAckaÝ-Dick, J., Reid, L. and RidDell, R. W. (1959). Thorax. 14, 104.

Eade, A. W. T., Harrison, G. K., Large, S. E. and Mackay-Dick, J. (1968). J. roy, Army med. Cps. 114,24

Foster-Carter, A. F., Myers, M., Goddard, D. O. H., Young, F. H. and Benjamin, B. (1952). Brompton Hosp. Rep. 21, 1.

Gangadharam, P. R. J., Devadatta, S., Fox, W., Narayanan, N. C. and Selkon, J. B. (1961). Bull. Wld Hlth Org. 25, 793.

Grumbach, F., Rist, N. and Riebel, J. (1952). Ann. Inst. Pasteur. 83, 397.

LARGE, S. E. and Dimond, A. D. (1964). Tubercle (Lond.). 45, 336.

LEADING ARTICLE. (1958), Lancet, ii, 1053.

LEADING ARTICLE, (1960). Lancet, ii, 639.

MACKAY-DICK, J. (1954). Lancet, ii, 44.

MACKAY-DiCK, J. (1958). N.A.P.T. Commonwealth Chest Conference Transactions, London.

MaCKAY-Dick, J. (1960). J. roy. Army med. Cps. 106, 93.

MACKAY-Dick, J. (1962). Tubercle (Lond.). 43, 461.

MaCKAY-Dick, J. (1963a). J. roy. Army med. Cps. 109, 82.

MACKAY-DiCK, J. (1963b). J. roy. Army med. Cps. 109, 225.

MACKAY-DiCK, J. (1963c). Lancet. i, 1429.

MaCKAY-Dick, J. (1965). Brit. med. J. ii, 232.

Medical ReSEARCH COUNCIL. (1962). Tubercle (Lond.). 43, 201.

Mitchison, D. A. (1965). Brit. med. J. i, 161.

Tuberculosis Chemotherapy Centre, Madras. (1960). Bull. Wld Hlth Org. 23, 535.

TuberCulosis ChemotherapY CENTRE, MADRAS. (1963). Lancet $i$, 1078.

Tuberculosis Chemotherapy Centre, Madras. (1964). Bull Wld Hith Org. 31, 247.

Poole, G. and Stradling, P. (1965). Tubercle (Lond.). 46, 290.

C.B.

From the New Year Honours 1968

Major-General J. P. Douglas, O.B.E., Q.H.S., M.B., late R.A.M.C.

C.B.E.

Brigadier G. M. WARRACK, D.S.O., O.B.E., T.D., L.R.C.S., L.R.C.P., Res. of Off.

O.B.E.

Lieutenant-Colonel J. M. ADAM, B.Sc., M.B., Ph.D., R.A.M.C.

M.B.E.

Mr. J. H. GRUNDY, F.R.S.A., F.R.E.S.

R.R.C.

Colonel B. M. Robertson, A.R.R.C., Q.A.R.A.N.C.

Lieutenant-Colonel (Acting Colonel) J. M. ORFord, Q.A.R.A.N.C.

A.R.R.C.

Major Y. J. M. M. DunNing, Q.A.R.A.N.C.

\section{MITCHENER MEDAL}

The Mitchener Medal of the Royal College of Surgeons of England, has been awarded to Lieutenant-Colonel W. C. Moffatt, M.B., F.R.C.S., for 1968. 